\title{
EVALUACIÓN DE CARACTERÍSTICAS DE LAS PERSONAS MAYORES Y SU RELACIÓN CON LA CALIDAD PERCIBIDA DE LA GESTIÓN DEPORTIVA
}

\section{ASSESSMENT OF ELDERLY PEOPLE CHARACTERISTICS AND THEIR RELATIONSHIP WITH THE PERCEIVED QUALITY OF SPORT MANAGEMENT}

\author{
Onetti-Onetti, W. ${ }^{1}$ Castillo-Rodríguez, C. L. ${ }^{2}$ y Castillo-Rodríguez, A. ${ }^{3}$ \\ ${ }^{1}$ Onetti, W. Profesora en UNIR, Universidad Internacional de la Rioja. wanesa.onetti@unir.net \\ ${ }^{2}$ Castillo-Rodríguez, C. L. Estudiante de Grado de Medicina de la Universidad de Málaga. \\ carmencastle94@hotmail.com \\ ${ }^{3}$ Castillo-Rodríguez, A. Doctor en Educación Física y profesor del Departamento de Educación \\ Física y Deportiva. Universidad de Granada. acastillo@ugr.es
}

Código UNESCO: 6114 Psicología Social

Clasificación Consejo de Europa: 15. Psicología del Deporte

Correspondencia:

Castillo-Rodríguez, A.

acastillo@ugr.es

DOI: http://dx.doi.org/10.24310/riccafd.2018.v7i2.5095

\section{RESUMEN}

La gestión deportiva busca en todo momento una mayor fidelización y control de los usuarios que evalúan su servicio prestado a través de cuestionarios de calidad percibida. El objetivo de la presente investigación fue evaluar la calidad percibida en trescientas tres personas a través del Cuestionario de Evaluación de la Calidad Percibida en Servicios Deportivos (CECASDEP), validado en muestra española. Los resultados indicaron que la dimensión más valorada es la del profesor-monitor, aunque el resto de dimensiones (instalaciones deportivas, espacio de actividad, vestuarios y programas de actividades) también obtuvieron una puntuación elevada con respecto a los valores medios, lo que conlleva a relacionar la satisfacción con la calidad percibida, y por ende, la fidelización de los usuarios también aumenta, gracias en cierto modo a la motivación que le produce el servicio que le han prestado. Se concluye que la valoración del técnico deportivo es la más positiva, obteniendo un papel clave para todas las instituciones deportivas.

Palabras clave: calidad de servicios, evaluación de calidad, Calidad percibida, servicios deportivos, gestión deportiva. 


\section{ABSTRACT}

Sports management seeks at all times a greater loyalty and control of users who evaluate their service provided through questionnaires of perceived quality. The objective of the present investigation was to evaluate the perceived quality of three hundred and three people through the Questionnaire for the Evaluation of Perceived Quality in Sports Services (CECASDEP), validated in a Spanish sample. The results indicated that the most valued dimension is that of the teacher-monitor, although the rest of the dimensions (sports facilities, activity space, changing rooms and activity programs) also obtained a high score with respect to the average values, which leads to relate the satisfaction with the perceived quality, and therefore, the loyalty of users also increases, thanks in some way to the motivation that produces the service they have provided. It is concluded that the assessment of the sports technician is the most positive, obtaining a key role for all sports institutions.

Keywords: quality of services, quality evaluation, perceived quality, sports services, sports management.

\section{INTRODUCCIÓN}

La gestión del deporte se articula como un concepto que puede resultar confuso debido a la gran variedad de tareas o acciones que integra (1), siendo necesaria la implantación de adecuados planes de calidad buscando la evaluación en aras de la mejora (2).

La calidad percibida por las personas en su práctica de actividad física obliga a establecer estrategias basadas en la mejora de la satisfacción de los usuarios gracias a la incorporación de adecuados planes de calidad (3). Existen diversas herramientas que evalúan la calidad de los servicios (4-7). El deporte no sólo requiere de buenos técnicos, sino que precisa de nuevos elementos y técnicas, englobadas en torno al concepto gestión deportiva (8).

Muchos sistemas de evaluación de servicios existentes en la literatura científica demuestran que hay diferentes atributos que tienen directa o indirectamente relación con la lealtad del cliente (9). Dentro de estos modelos, Grönroos $(10,11)$ desarrolló un modelo tridimensional que sirvió de base para el modelo SERVQUAL propuesto por Parasuraman, Zeithaml y Berry (4), que ha sido el predominante en las últimas décadas a través de sus 5 dimensiones, pese a ser criticado fundamentalmente por Cronin y Taylor (12) que propusieron el modelo SERVPERF, así como en varias investigaciones que advirtieron de diferentes limitaciones $(7,13,14)$. Posteriormente, Dabholkar, Thorpe, y Rentz (15) propusieron un modelo multinivel de calidad de servicio mientras que el modelo de Brady y Cronin (16) concibe la calidad del servicio a través de 3 dimensiones principales. 
Por tanto, el objetivo de este estudio fue comprobar el grado de satisfacción de los usuarios mayores de 40 años de distintos centros deportivos de la ciudad de Málaga.

\section{MATERIAL Y MÉTODO}

\section{Participantes}

En el presente estudio han participado 303 usuarios (123 hombres y $180 \mathrm{mu}$ jeres) de centros deportivos de la ciudad de Málaga, donde la gestión es exclusivamente municipal. El lugar de entrenamiento de estos participantes son 3 centros deportivos, distribuidos en zonas diferentes de la ciudad, donde se encuentran instalaciones deportivas municipales de características similares. El estudio fue realizado de acuerdo con la Declaración de Helsinki y la ley orgánica 15/1999, de 13 de diciembre, de protección de datos de carácter personal.

Los usuarios asisten 2 ó 3 veces en semana al centro deportivo, realizan una actividad dirigida con técnicos y no son usuarios de actividad libre y esporádica, lo que ofrece fiabilidad y validez de las respuestas. Los criterios de inclusión del estudio fue ser mayor de 40 años y asistir asiduamente a un centro deportivo de la ciudad de Málaga.

\section{Instrumentos}

Partiendo de los estudios realizados por Gronroos (10), Gálvez y MoralesSánchez (3), formulan un cuestionario de Evaluación de la Calidad Percibida en Servicios Deportivos (CECASDEP), creando una herramienta de evaluación dentro del ámbito de la gestión deportiva que profundiza en las necesidades y demandas de los usuarios, teniendo en cuenta tanto los aspectos tangibles e intangibles del servicio y basaron su evaluación en 6 bloques de cuestiones: instalaciones deportivas, atención al usuario, espacio de actividad, vestuarios, programas de actividades y profesor-monitor.

El cuestionario inicial lo conformaban 71 ítems distribuidos en las 6 dimensiones. Posteriormente, Gálvez-Ruiz y Morales Sánchez (3) modifican el cuestionario agrupando preguntas, acortando bloques, quedando en 5 dimensiones (instalaciones deportivas, espacio de actividad, vestuarios, programas de actividades y profesor-monitor) y 51 ítems, que configuran el definitivo cuestionario. Se ha demostrado su validez y fiabilidad para la población española (fiabilidad entre 0.70 y 0.96 en todas las dimensiones), con valores muy aceptables según Nunnally (17).

La respuesta a las cuestiones se puntúa a través de escala Likert entre 1 y 5 siendo el valor 1 totalmente en desacuerdo y el valor 5 para totalmente de acuerdo. 


\section{Procedimiento}

La recogida de datos fue realizada en febrero de 2013. Todos los participantes aceptaron voluntariamente a participar en este estudio, tras la explicación del objetivo del mismo. Se contó además con el consentimiento de la institución deportiva, recogiendo los datos de forma individual en los espacios en los que se impartían los diferentes programas de actividad física, con presencia de los investigadores para proporcionar la ayuda necesaria cuando fue requerida para cumplimentar los cuestionarios correctamente. Este estudio fue aprobado por el comité de ética de la Universidad de Málaga.

\section{Análisis estadístico}

El tratamiento informático estadístico se ha realizado en SPSS for Windows (SPSS Inc). La puntuación entre 1 y 5 del cuestionario nos permite calificar el punto medio en el 2.5, siendo los resultados por encima de esta media como aprobados y las notas por debajo de la media de 2.5 puntos, suspenden.

Se ha comprobado la no normalidad de las variables, utilizando en consecuencia, tests no paramétricos. Se han realizado análisis descriptivos, comparativos y correlacionales entre las variables del estudio. El nivel de significación establecido fue de $P<0.05$.

\section{RESULTADOS}

En primer lugar, se puede observar la percepción de la calidad de los servicios deportivos en función del género (Tabla 1). En la misma, se observan diferencias significativas en todas las dimensiones $(P<0.05)$. Se puede apreciar que el nivel de satisfacción en las mujeres es mayor en todas las dimensiones encuestadas.

\begin{tabular}{lllllll}
\hline & SEXO & $\mathrm{N}$ & Media & $\mathrm{DT}$ & $\mathrm{T}$ & $\mathrm{P}$ \\
\hline Instalación-Municipal-Deportiva & 1 & 123 & 3,43 & 0,47 & $-4,188$ & 0,000 \\
\cline { 2 - 8 } & 0 & 180 & 3,65 & 0,45 & & \\
\hline Espacios-Deportivos & 1 & 123 & 3,01 & 0,36 & $-6,234$ & 0,000 \\
\hline & 0 & 180 & 3,29 & 0,40 & & \\
\hline Vestuarios & 1 & 123 & 3,08 & 0,37 & $-6,209$ & 0,000 \\
\hline Programa-Actividades & 0 & 180 & 3,37 & 0,43 & & \\
\hline Técnico-Deportivo & 1 & 123 & 3,46 & 0,40 & $-5,867$ & 0,000 \\
\hline & 0 & 180 & 3,73 & 0,39 & & \\
\hline
\end{tabular}

Sex: 1: men; 2: women.

Tabla 1. Test U Mann Whitney de las variables de calidad de los servicios deportivos en función del género. 
A continuación se ha realizado análisis comparativo en función de los años del usuario con respecto a la percepción sobre la calidad del servicio (Tabla 2). En este sentido, los usuarios con 2 años en la instalación perciben de forma inferior la calidad prestada por el servicio deportivo que los usuarios con 4 años de fidelización $(P<0,05)$.

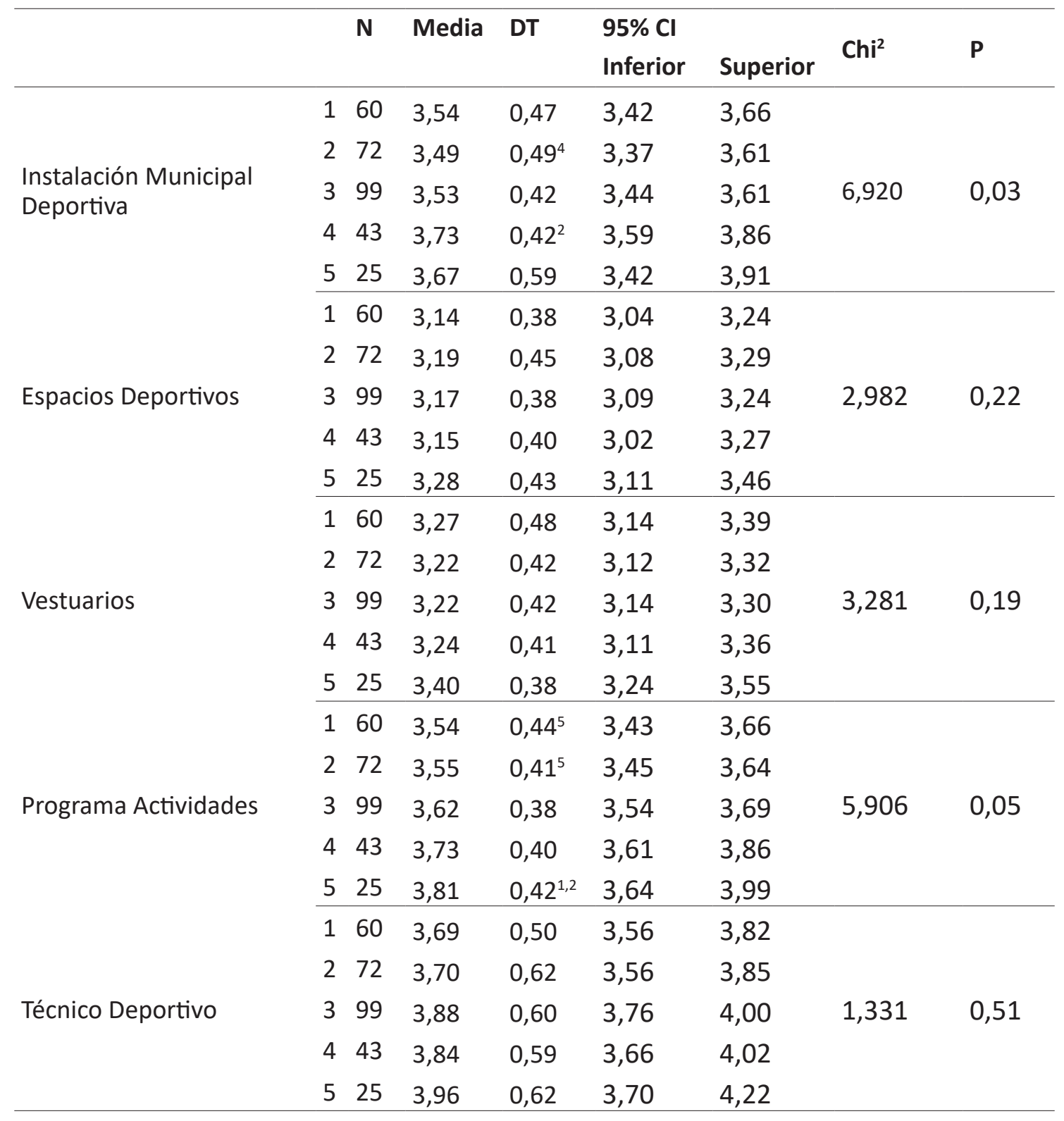

TABLA 2. Prueba de Kruskal-Wallis en función a los años de fidelización (1-5).

Posteriormente, se ha realizado un análisis de correlación entre las dimensiones del test (Tabla 3). Se observa que las dimensiones de la percepción de la calidad de los servicios deportivos, se correlacionan positivamente entre ellas. Destacar que la percepción de la calidad prestada por el técnico deportivo se relaciona positivamente con la percepción de la calidad hallada en vestuarios y programas de actividades (Rho: 0,53 y $0,56, P<0,01$ respectivamente). 


\begin{tabular}{lllll}
\hline & $\begin{array}{l}\text { Espacios } \\
\text { Deportivos }\end{array}$ & Vestuarios & $\begin{array}{l}\text { Programa } \\
\text { Actividades }\end{array}$ & $\begin{array}{l}\text { Técnico } \\
\text { Deportivo }\end{array}$ \\
\hline Edad & $0,169^{* *}$ & $0,137^{*}$ & $0,229^{* *}$ & $0,307^{* *}$ \\
\hline Instalación-Municipal-D & $0,442^{* *}$ & $0,345^{* *}$ & $0,445^{* *}$ & $0,322^{* *}$ \\
\hline Espacios-Deportivos & & $0,672^{* *}$ & $0,513^{* *}$ & $0,511^{* *}$ \\
\hline Vestuarios & & & $0,476^{* *}$ & $0,533^{* *}$ \\
\hline Programa-Actividades & & & & $0,563^{* *}$ \\
\hline$* * P<0,01 ;{ }^{*} P<0,05$ & & & &
\end{tabular}

Tabla 3. Test Rho de Spearman entre las variables de percepción de calidad de los servicios deportivos

\section{DISCUSIÓN}

El objetivo de este estudio fue comprobar la satisfacción de los usuarios de la ciudad de centros deportivos.

La calidad es fundamental para el éxito y el logro de resultados y en consecuencia, la fidelización del usuario. Esto debe considerarse como un valor que abarca a toda su organización y donde se produce una interacción constante entre todos los elementos que intervienen en el proceso (18). Y como eje de este proceso, el usuario y su opinión sobre la calidad percibida son fundamentales en la evaluación de satisfacción $(19,20)$.

En la actualidad, la investigación sobre la calidad de los servicios en el sector deportivo se está incrementando y debe ser controlada para la fidelización de usuarios y el mantenimiento de estándares de calidad que permitan su comparación con otros entornos deportivos (7). Hasta la fecha la mayoría de los estudios de calidad se han centrado en identificar determinadas dimensiones en determinadas actividades deportivas tanto de ocio como de espectáculo, y aunque los investigadores han sugerido diversas estructuras para compendiar los factores, aún no hay un acuerdo general que contemple todas las dimensiones.

Llevar a cabo una evaluación periódica y fiable de la calidad percibida de los usuarios de los servicios que prestan los centros deportivos no es fácil. En este estudio hemos aplicado el Cuestionario CECASDEP desarrollado y validado por Gálvez y Morales-Sánchez (3).

Existen otros instrumentos que son válidos para evaluar la calidad percibida de los usuarios de servicios deportivos tanto a nivel nacional como internacional. Entre los más destacados encontramos en orden cronológico las aportaciones para evaluar la calidad percibida de Grönroos (10), Cronin y Taylor (12), Teas (21) y Brady y Cronin (16), pero sin duda son los trabajos de Parasuraman, Zeinthaml y Berry (4) con la Escala SERQVUAL, los que obtuvieron mayor repercusión. Esta escala basada en las expectativas, es la herramienta más utilizada en los estudios de evaluación de calidad percibida de los servicios (22) 
Kim y Kim (14) desarrollan la Escala QUESC (Quality Excellence of Sports Centres) como instrumento específico para evaluar la calidad en Corea donde establecen once dimensiones (ambiente, actitud de los empleados, fiabilidad, información, programa de actividades, consideración personal, privilegios, precios, facilidad de comprensión, estimulación y comodidad). En España, destacar el Inventario de Calidad en Programas de Actividad Física (ICPAF) de Hernández Mendo (23) que busca ser una herramienta útil de medida en la evaluación de los aspectos relevantes de la actividad física midiendo la satisfacción de los usuarios (24).

Vila, Sánchez y Manassero (25) evalúan la satisfacción percibida por los usuarios en una encuesta adaptada por la Universidad de Valencia e Islas Baleares para medir la satisfacción de clientes y Rial et al. (26) que presentan la Escala QSport-10 con el objetivo de medir la calidad percibida en los centros deportivos privados a través de un cuestionario compuesto por 10 ítems estructurado en dos dimensiones: personal e instalaciones.

En estos estudios resaltan la importancia del técnico de la organización. En el presente estudio, los participantes califican con una puntuación mayor a los técnicos deportivos con hasta 4.05 puntos sobre 5 (tabla 1). En la misma línea se encuentra el estudio de Dorado (27), el cual, determina el elemento mejor valorado el personal y el peor, las instalaciones deportivas.

Un gran hallazgo se ha comprobado en este estudio. Los usuarios con mayor número de años de fidelización, realizan puntuaciones más altas (tabla 2). No se ha encontrado literatura científica sobre esta relación hasta la fecha. Esta puntuación puede estar supeditada a unos factores de bienestar y confianza con la instalación en general, aunque parece tener relación con un mayor enlace con el técnico, lo cual, debe analizarse en el futuro para establecer conclusiones más firmes.

\section{CONCLUSIONES}

La calidad percibida es clave en los modelos de gestión actuales y en las demandas de los clientes de nuestros servicios. A través del cuestionario CECADESP se ha conseguido demostrar que el grado de satisfacción general de la calidad percibida de los usuarios mayores de 40 años de los centros deportivos municipales de la ciudad de Málaga, se puede catalogar muy positivo, con una puntuación de 7.1 sobre 10.

Además han valorado muy positivamente los recursos humanos de los que dispone la organización, creando lazos afectivos. Las mujeres tienen una satisfacción superior con el centro, se acomodan más a las circunstancias y son más fieles al centro deportivo; mientras los hombres son más demandantes, menos acomodado y solicitan cambios de programas en las actividades. 


\section{REFERENCIAS}

1. Molina-García J, Castillo I. Pensamiento sobre la gestión deportiva pública: un estudio cualitativo con universitarios valencianos. Revista Actividad Física y Deporte: Ciencia y Profesión. 2009;10:13-24.

2. García J, Cepeda G, Martín D. La satisfacción de clientes y su relación con la percepción de calidad en Centro de Fitness: utilización de la escala CALIDFIT. Revista de Psicología del Deporte. 2012;21(2):309-19.

3. Gálvez Ruiz P, Morales Sánchez V. Evaluación de la calidad en los programas municipales deportivos: generalizabilidad y optimización de diseños de medida. Cuadernos de Psicología del Deporte. 2011;11(2):123-30.

4. Parasuraman A, ZeithamI VA, Berry LL. SERVQUAL: A multiple-item scale for measuring consumer perceptions of service quality. Journal of Retailing. 1988;64:12-40.

5. Afthinos $Y$, Theodorakis ND, Nassis P. Customer's expectations of service in Greek fitness centres. Gender, age, type of sport center and motivation differences. Managing Service Quality. 2005;15(3):245-58.

6. Sanz I, Redondo J, Gutiérrez P, Cuadrado G. La satisfacción en los practicantes de spinning: elaboración de una escala para su medición. Motricidad: European Journal of Human Movement. 2005;71(13):17-36.

7. Calabuig F, Quintanilla I, Mundina JJ. La calidad percibida de los servicios deportivos: Diferencias según instalación, género, edad y tipo de usuario en servicios náuticos. International Journal of Medicine and Science of Physical Activity and Sport. 2008;4(10):25-43.

8. Mestre JA. Componentes de la gestión deportiva. Una aproximación. Revista de Educación Física. 2013;2(2):1-19.

9. Theodorakis ND. A cross-validation study of the other customers perceptions scale in the context of sport and fitness centres. RICYDE. Revista Internacional de Ciencias del Deporte. 2014;35:63-74

10. Grönroos C. A service quality model and its marketing implication. European Journal of Marketing. 1984;18:36-44.

11. Grönroos C. Service quality: The six criteria of good perceived service Review of Business. 1988;9:10-3.

12. Cronin JJ, Taylor SA. Measuring service quality: A reexamination and extension. Journal of Marketing. 1992;56:55-68.

13. Alexandris K, Zahariadis $P$, Tsorbatzoudis C, Grouios G. An empirical investigation of the relationships among service quality, customer satisfaction and psychological commitment in a health club context. European Sport Management Quarterly. 2004;4:36-58.

14. Kim D, Kim SY. QUESC: An instrument for assessing the service quality of sport centres in Korea. Journal of Sport Management. 1995;9:208-20.

15. Dabholkar P, Thorpe DI, Rentz JO. A measure of service quality in retail stores: Scale development and validation. Journal of the Academy of Marketing Science. 1996;24:316.

16. Brady M, Cronin J. Some new thoughts on conceptualizing perceived service quality: A hierarchical approach. Journal of Marketing. 2001;65:34-49. 
17. Nunnally JR, Marion Diehl. Loading apparatus for round bales. U.S. Patent No 3,941,265, 2 Mar. 1976.

18. García Ferrando M. La gestión del deporte en el ámbito municipal: de la promoción a la fidelización del cliente. In M. Latiesa et al. (comp.). Deporte y cambio social en el umbral del siglo XXI, 17-40. Madrid: Esteban Sanz, 2001.

19. García-Mas A. Psicología del turismo deportivo. En A. Hernández Mendo (Coord.), Psicología del Deporte (Vol. III): Aplicaciones, 2, 6-24. Buenos Aires. Tulio Guterman, 2003.

20. Redondo JC, Olivar D, Redondo A. El modelo EFQM de gestión de la calidad en las instalaciones deportivas. Sevilla: Wanceulen, 2006.

21. Teas RK. Expectations, performance, evaluation and consumer's perceptions of quality. Journal of Marketing. 1993;57:18-34.

22. Morales Sánchez V, Hernández Mendo A, Blanco A. Evaluación de la calidad en organizaciones deportivas: adaptación del modelo SERVQUAL. Revista de Psicología del Deporte. 2009;18(2):137-50.

23. Hernández Mendo A. Un cuestionario para evaluar la calidad en programas de actividad física. Revista de Psicología del Deporte. 2001;10(2):179-96.

24. Hayes BE. Cómo medir la satisfacción del cliente. Desarrollo y utilización de cuestionarios. Barcelona: Ediciones Gestión 2000, 1995.

25. Vila I, Sánchez C, Manassero MA. Satisfacción percibida de los usuarios de las instalaciones deportivas municipales de Palma de Mallorca. Revista Iberoamericana de Psicología del Ejercicio y el Deporte. 2009;4(1):59-74.

26. Rial J, Varela J, Rial A, Real E. Modelización y medida de la Calidad Percibida en centros deportivos: la escala QSport-10. Revista Internacional de Ciencias del Deporte. 2010;18(6):57-73.

Dorado, A. Análisis de la satisfacción de los usuarios del servicio deportivo municipal. Revista de Educación Física INDEref. Avaliable in: http://www.inderef.com/content/view/35/113, 2007.

Referencias totales citadas: 27

Referencias citadas correspondientes a la Rev Ib CC Act Fis Dep: 0 